\section{Minimally-invasive procedure for pelvic leak points in women}

\author{
Roberto Delfrate, Massimo Bricchi, \\ Claude Franceschi \\ Surgical Unit, Figlie di San Camillo \\ Hospital, Cremona, Italy
}

\begin{abstract}
Pelvic leak points (PLP) may be responsible for vulvar, perineal and lower limb varicose veins, in women during and/or after pregnancy. The accurate anatomical and hemodynamic assessment of these points, the perineal (PP), inguinal (IP) and clitoral points $(\mathrm{CP})$ and their surgical treatment under local anesthetics as defined by Claude Franceschi is a new therapeutic option. The aim of this study was to assess the reliability and durability of the PLP reflux ablation using a minimally-invasive surgical disconnection at the PLP level in women with varicose veins of the lower limbs fed by the PLP. In this open-label trial 273 pelvic leak points free of pelvic conges-
\end{abstract} tion syndrome, with at least a 12-month follow-up, were assessed. 273 PLP treated: PP $(n=177)$, IP $(n=91)$ and CP $(n=5)$. Followup: Period $=12$ to 92 months (mean $=30.51$ months). Age from 29 to 77 years (mean=45). The only 3 patients over 70 years $(71,74,77)$ showed a high-speed reflux from a I point that fed symptomatic varicose veins of the lower limb. Exclusion criteria: pelvic congestion syndrome, BMI $>24$, venous malformations, a post thrombotic varicose vein. Diagnosis was performed using echo duplex and PLPs selected for treatment when refluxing at Valsalva + Paraná + squeezing maneuvers. A surgical skin marking of the PLP had been performed using echo duplex before surgery. Surgery consisted of minimally invasive dissection and selective division and ligation with non-absorbable suture of the refluxing veins and fascias at the PP, IP and $\mathrm{CP}$ pelvic escape points, under local anesthesia in a single center. The follow-up consisted of an echo duplex ultrasound, searching for reflux at the PLP treated thanks to the Valsalva maneuver, within 2 weeks, after 6 and 12 months and then yearly. The main endpoint of the study was the immediate elimination of the reflux at the PLP treated. The second endpoint was the long-term durability of the reflux ablation at the PLP treated. $267(97.8 \%)$ without PLP reflux redo. 6 (2.2\%) PLP reflux recurrences $(\mathrm{PP}=4, \mathrm{IP}=1, \mathrm{CP} 1) .3$ patients with PLP reflux recurrence undergo a redo sur- gery $(1.1 \%)$ where $\mathrm{PP}=2(0.7 \%)$ and $\mathrm{IP}=1$ $(0.3 \%)$.

This study shows the feasibility and durability of reflux ablation at the PLP level thanks to a minimally-invasive surgical treatment of the PLP and it demonstrates that there is no need for pelvic varicose embolization in patients without clinical signs of pelvic congestion syndrome. The accurate ultrasound assessment of each specific pelvic leak as well as a special surgical technique (vein division, non-absorbable suture of veins and fascias) seems to be the key for satisfactory outcomes.

\section{Introduction}

Pelvic leak points (PLP) reflux may be responsible for vulvar and/or lower limb varicose veins, as defined by Claude Franceschi. ${ }^{1-5}$ PLP treatment represents $8.3 \%$ of all the CHIVA treatment we performed. According to the CHIVA strategy the treatment of all the escape points is mandatory, and possible through a minimally-invasive surgical treatment as an alternative to parietal sclerotherapy and pelvic veins embolization.

Several studies $^{6-9}$ based on various assessments (clinical, ultrasound, venography) consider around $10 \%$ of pelvic leaks in women with varicose veins. It has been reported in literature that the number pelvic leaks in multiparous are 4 times more frequent than in nulliparous. ${ }^{7}$ Particularly the recurrences after stripping were related to pelvic venous reflux and evaluated as $17 \%$ but not specifically anatomically defined and not specifically treated. ${ }^{10,11}$ Beside the obturatory and gluteal refluxes, Claude Franceschi, thanks to Duplex Ultrasound Scan (DUS), detected three different main pelvic leak points (PLP) responsible for varicose veins of the lower limbs in mono and multiparous women..$^{1-5}$ These PLP are called perineal points (PP), inguinal points (IP) and clitoral points (CP) as represented in Figure 1A. A perineal point (PP) is the superficial perineal vulvar fascia hole crossed by the perineal vein that collects the posterior labial vein, then connects to the internal pudendal vein. The perineal vein drains the skin of the perineum, then receives the posterior labial veins and passes through the superficial aponeurosis of the perineum (fascia perinalis) by way of an orifice that we call PP. ${ }^{12-14} \mathrm{After}$ crossing the PP, the vein ascends with bulbar and cavernous veins to the pudenal vein in the Alcock's canal. In case of leakage, reflux follows the same pathway in the opposite direction. Reflux can not only cause dilatation of the labial and perineal veins but also
Correspondence: Roberto Delfrate, Surgical Unit, Figlie di San Camillo Hospital, via Fabio Filzi 56, 26100 Cremona, Italy. E-mail: roberto.delfrate@icloud.com

Key words: Pelvic leak points; inguinal point; perineal point; clitoral point.

Received for publication: 28 August 2018. Revision received: 28 January 2019.

Accepted for publication: 30 January 2019.

This work is licensed under a Creative Commons Attribution 4.0 License (by-nc 4.0).

(C) Copyright R. Delfrate et al., 2019

Licensee PAGEPress, Italy

Veins and Lymphatics 2019; 8:7789

doi:10.4081/vl.2019.7789

extend to the ipsilateral saphenous network through either perineal-to-labial and perineal-to-external pudendal vein anastomoses or through any other incompetent vein in between. It can also feed a contralateral varicosity through labiolabial and perineoperineal anastomoses. Reflux in the medial pudendal vein is itself fed either actively or potentially by any constitutionally incontinent ipsilateral and contralateral upstream genital, visceral iliac, and ovarian vein and by the inferior vena cava. An Inguinal Point (IP) is the superficial inguinal annulus crossed by the mons veneris veins that connects to the uterine round ligament vein. Reflux of the round ligament vein of the uterus can feed vulvar (labial) and perineal varicose veins and lower extremity varicose veins via residual branches of the Nuck's canal that reflux directly or indirectly to the subcutaneous abdominal, external pudendal, superficial dorsal of the clitoris, and labial veins, then possibly toward varices in the saphenous network. Once again, reflux in the round ligament vein is itself fed either actively or potentially by any constitutionally incontinent ipsilateral and contralateral upstream genital, visceral, iliac, or ovarian vein and by the inferior vena cava. A CP is the anastomotic plexus between the bulbar vein and superficial dorsal clitoris that connects to the medial pudendal vein. ${ }^{15}$ Reflux can feed ipsilateral or contralateral perinal and anterior labial veins and or the lateral pudendal then the GSV (Figure 1B). As the PLP reflux is usually fed by hypogastric tributaries and/or ovarian incompetent veins, and the PLP reflux occurs as a result of recurrence after conventional stripping, some authors suggest embolization of these veins as a first step treatment of peripheral varicose veins of the lower limbs. ${ }^{16,17}$ In this 
study, the first step in treatment was surgical ablation of the PLP reflux in patients free of pelvic congestion. PLPs represent the escape point of the shunts type 4 and 5 , according to the Teupitz CHIVA classification. ${ }^{18}$ These types of shunts are characterized by an escape point external to a saphenous axis with a direct re-entry point located on a saphenous axis in the type 4, and indirect re-entry point in type 5 .

\section{Materials and Methods}

\section{Study design}

In this open-label trial a group of 273 PLPs treated with at least a 1 -year followup were evaluated: PP ( $n=177)$, IP ( $n=91)$ and $\mathrm{CP}(\mathrm{n}=5)$. The follow up period varied from 12 to 92 months (mean=30.51 months). Age ranged from 29 to 77 years (mean=45). The group included 3 patients over 70 years $(71,74,77)$ as they showed a high-speed reflux from a I point that fed symptomatic varicose veins (CEAP 4) of the lower limb. CEAP classification 2-4: visible varicose veins, or painful varicose veins, itch, oedema, skin pigmentation. The exclusion criteria were the evidence of a pelvic congestion syndrome, $\mathrm{BMI}>24$; furthermore, all patients with congenital varicose veins due to venous malformations, varicose veins secondary to prior deep vein thrombosis, associated systemic pathologies, those who refused surgical treatment, who could not participate in long-term follow-up or had given birth less than 9 months previously were excluded from the study. A written informed consent was provided to all the patients. The study was conducted by the same surgical team who performed ultrasound diagnosis, mapping and pre-operatory marking as well as the procedures. The diagnosis was assessed with Duplex Ultrasound Scan (DUS) by the surgeons thanks to a $10-18 \mathrm{MHz}$ linear probe. The PRF was set between 0.75 and $1 \mathrm{KHz}$, capable of detecting even low-speed reflux from 0.05 to $0.10 \mathrm{~m} / \mathrm{s}$.

\section{Aim of the study}

This study aimed to assess the immediate elimination and the long term persistence of pelvic reflux ablation at the PLP treated with a minimally invasive surgical operation under local anesthesia, in uniparous or multiparous women, free of pelvic congestion syndrome (chronic pelvic pain and continuous reflux at the PLP level without any breath modulation, in standing and supine position), regardless of the degree and configuration of the varicosities. The PLP reflux was partly (existence of other escape points) or totally responsible for lower limbs varicose veins.

\section{PLP diagnosis and description}

Clinical data allow diagnosis of vulvar and perineal varices but cannot determine the leak point, since leakage from I, P or C points lead to the same clinical manifestations. A full color duplex scan allows precise identification of $\mathrm{I}, \mathrm{P}$ and $\mathrm{C}$ points. The specific criteria of reflux at the PLP was a Valsalva descending flow provoked by having the women blow into a blocked straw while standing. ${ }^{19}$ Conversely, the diastolic

$\mathbf{A}$

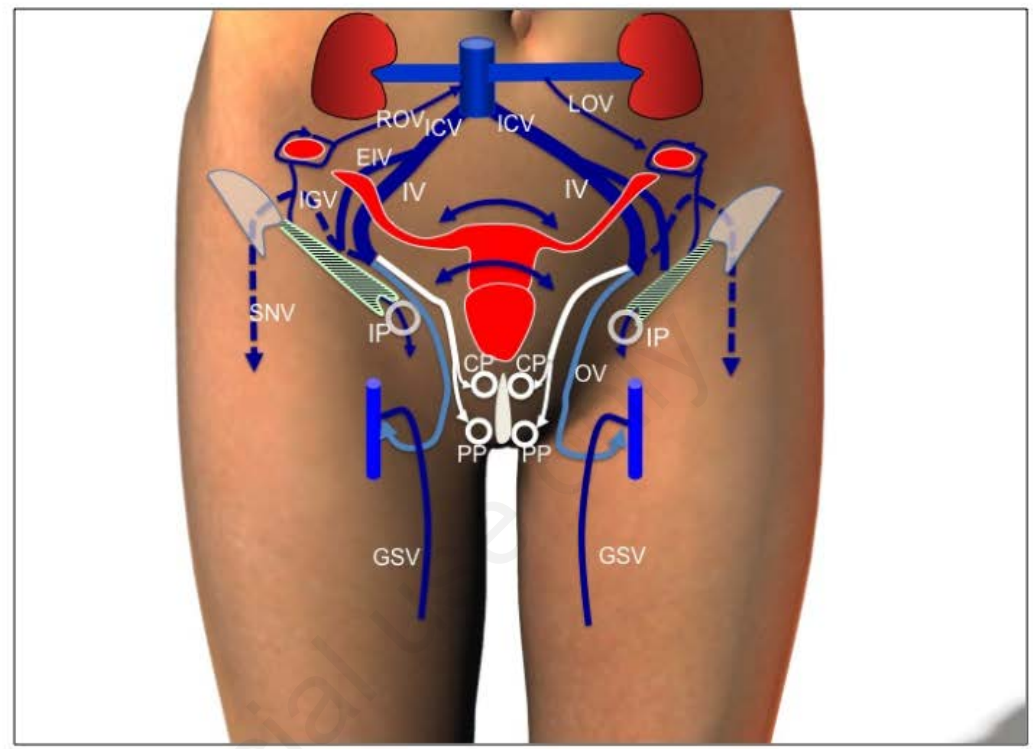

\section{B}

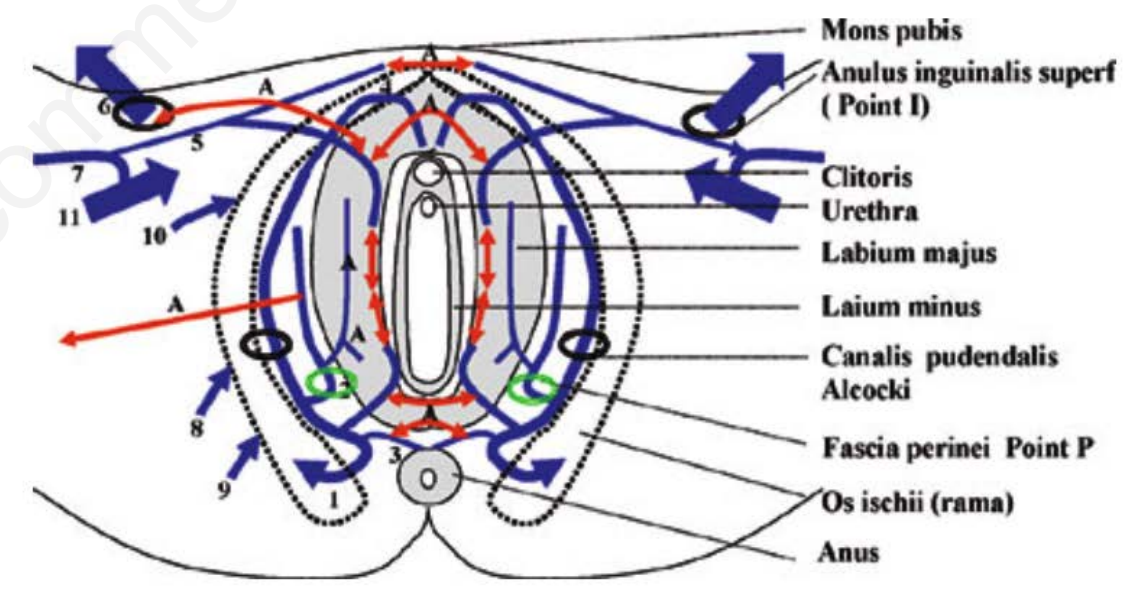

Figure 1. A) Frontal view of the pelvis passing through the perineal and inguinal leak points (points PP, CP and IP). Note the number of anastomoses. LOV (left ovarian vein); ROV (right ovarian vein); ICV (iliac common vein); IV (internal iliac vein); EIV (external iliac vein); the white lines represented the internal pudendal veins feeding the $C P$ (clitoral points) and the PP (perinea); GSV great saphenous vein; IP inguinal point, with the venous plexus of the round ligament passing through the superficial ring of the inguinal canal; OV (obturator veins) feeding the medial muscular circumflex vein; IGV inferior gluteal veins an veins of the sciatic nerves SNV; B) Perineal view showing the perineal and inguinal leak points (points $P$ and $I$ ). Note the number of anastomoses (A). 1 , internal pudendal vein; 2 , perineal vein; 3 , vena rectalis inferior (inferior rectal vein); 4 , vena bulbi vestibuli et clitoridi (vein of bulb of vestibule and clitoris); 5 , vena pudenda externa (external pudendal vein); 6, ligamentum teres uteri vena (round ligament vein of the uterus); 7, vena saphena magna (great saphenous vein); 8 , gluteal vein; 9, sciatic vein; 10 , obturator vein; 11, vena femoralis (femoral vein). 


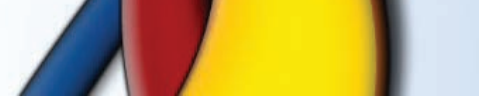

flow at the descending tributaries of the great saphenous veins (GSV) arch evoked by calf squeezing or Paraná maneuver is not specific and may be present in the absence of refluxing PLP, so it is a source of false positives. Continuous reflux at rest (without any dynamic maneuver) may be due to a collateral compensatory draining flow caused by a downstream obstacle and thus prohibits any disconnection. The patient's evaluation was completed by an exhaustive ECD in order to achieve a complete hemodynamic mapping of the venous insufficiency. With the patient in a standing position, IP can be located approximately 1 to $3 \mathrm{~cm}$ above the femoral vein and just medially to the epigastric veins and CP medially to the SFJ towards the clitoris. The reflux is seen outwards throughout the inguinal canal (Figure 2). PP is generally located at the junction of the posterior fourth and anterior three-fourths of the labia majora. It can be detected if the patient is in a standing position with her foot resting on a platform 20 $\mathrm{cm}$ high or in a lithotomy position for a transperineal ultrasound (not intravaginal because the probe imaging would be too deep compared to the superficial PP). The reflux activated by Valsalva maneuver induces backflow from the Alcock's canal to the perineal and labial veins (Figure 3). The Alcock's canal is located medially to and just above the ischiopubic branch.

\section{Interventions}

The patients were ambulatory. The same surgeon performed the marking and intervention. The anesthesia was local: less than $3 \mathrm{ml}$ of a mixture of lidocaine $(2 \%)$ and ropivacaine $(7.5 \mathrm{mg} / \mathrm{dL})$. Incision according to skin marking: from 10 to $14 \mathrm{~mm}$ (Figures 4 and 5). Effective treatment of lower extremity superficial venous reflux of pelvic origin can only be achieved by ligation of the leak points in the same way as is necessary to ligate a refluxing perforating vein or junction. Proximal or distal ligation without ligation at PP and/or IP level will be followed by recurrence due to collateral flow. The perineal and inguinal leak points (points $\mathrm{P}$ and I) act as perforating veins (Figure 6). Remote disconnection (B, C) invariably fails either immediately or secondarily because of the presence of many branches and anastomoses (Figure 7). Perineal and genito-crural nerves were preserved respectively at the PP and IP. Division and stump ligation of the refluxing vein with non-absorbable braided coated suture and additional polypropylene 6 zero monofilament transfixed suture for the PP, and 4 zero for the IP. Furthermore, the stump of round ligament was positioned and overturned inside the inguinal canal,
$\mathbf{A}$

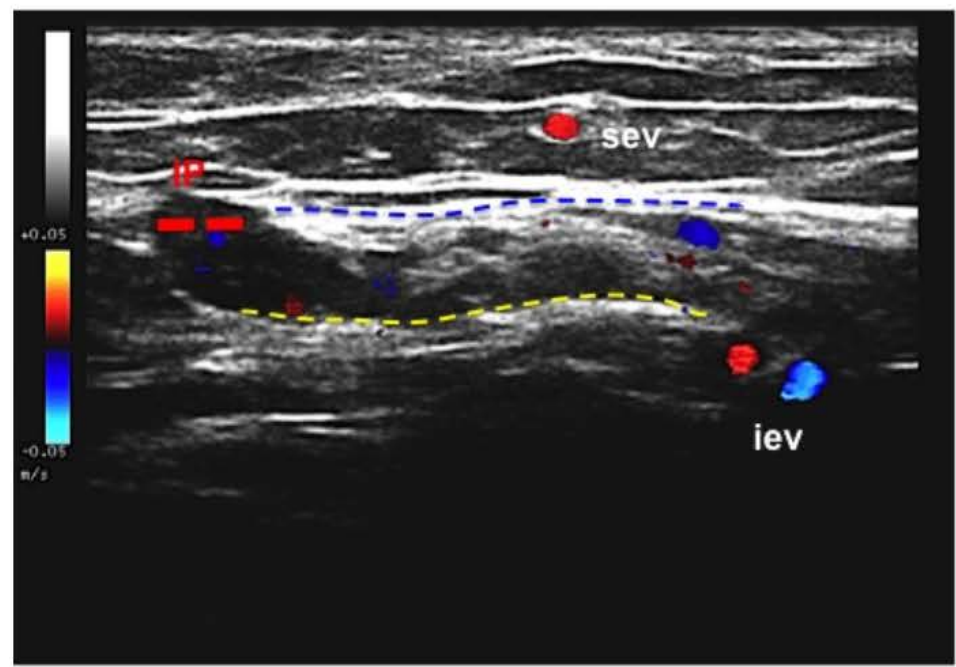

B

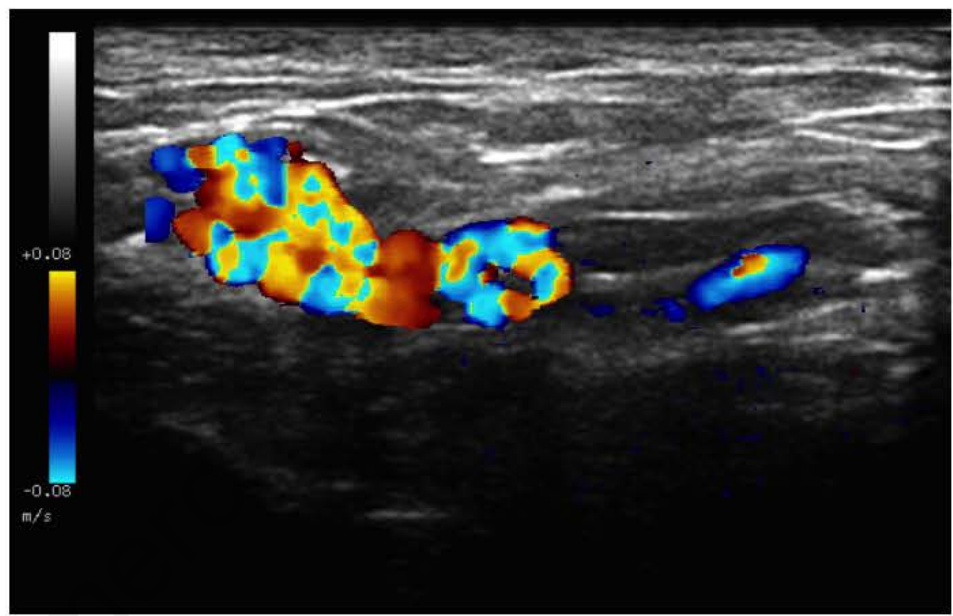

C

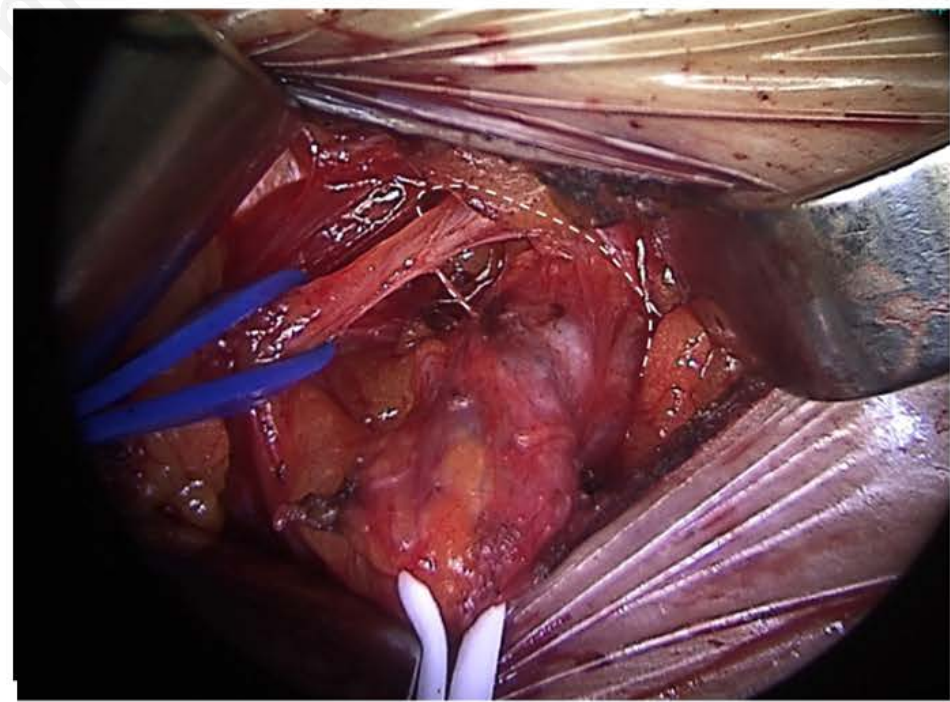

Figure 2. A) The venous plexus of the round ligament in the inguinal canal, its relationship with the inferior epigastric vessels, the subcutaneous ring (red dashed line) is the IP through which a pelvic reflux is transmitted during Valsalva to the lower limbs. The external oblique muscle fascia is the hyperechoic line above the plexus (blue dashed line), while the hyperechoic line corresponding to the trasversalis fascia is clearly visible below it (yellow dashed lines); B) Valsalva maneuver. Clearly detectable reflux from the deep pelvic plexuses towards the surface through the inguinal canal and its superficial ring; $C$ ) Left IP dissection. The venous plexus of the round ligament on a white vessel loop. The genitocrural nerve on a blue vessel loop. 


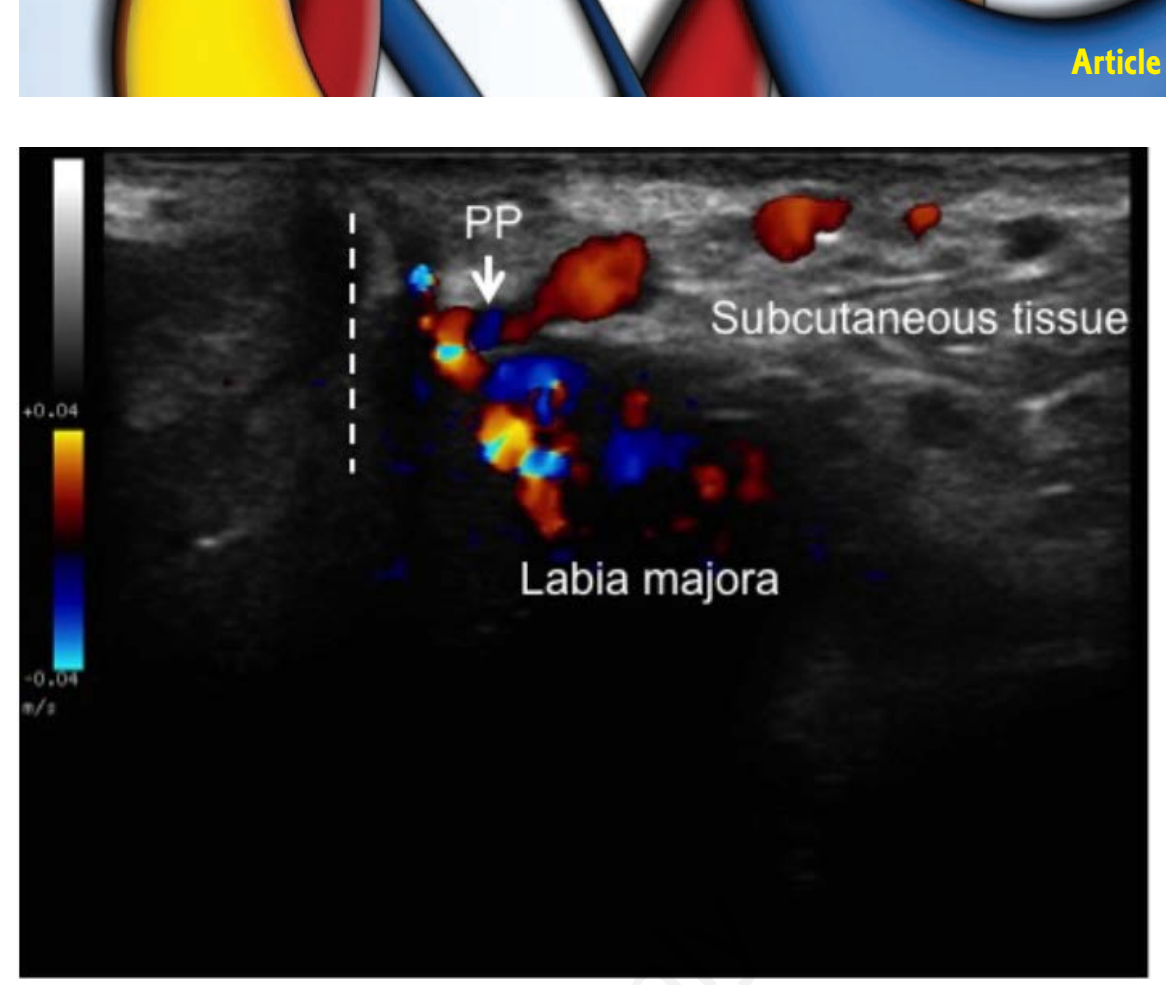
cy of the intervention was evaluated ing the Doppler response provoked by the Valsalva maneuver at the treated PLP. Reflux was considered a failure; no reflux was considered a success.

\section{Results}

Of the 273 PLP treated 267 (97.8\%) did not show any PLP reflux redo. A PLP reflux recurrence was detected in $6(2.2 \%)$ PLP reflux $(\mathrm{PP}=4, \mathrm{IP}=1, \mathrm{CP} 1) .3$ patients with PLP reflux recurrence undergo a redo surgery $(1.1 \%)$ where $\mathrm{PP}=2(0.7 \%)$ and $\mathrm{IP}=1$ $(0.3 \%)$. In the remaining 3 patients with reflux redo sclerotherapy was proposed. No deep vein thrombosis, pulmonary thromboembolism, or death occurred. Considering possible surgical complications, no bruises, subcutaneous inguinal or perineal hemorrhage, saphenous nerve neuralgia, wound infection or superficial phlebitis occurred; there was only one inguinal bleeding that needed an immediate surgical exploration of the inguinal canal and hemostasis thanks to a vessel legation: after this clinical case the ligation technique has been modified and currently we do a double ligation with a non-absorbable braided coated suture and a transfixed polypropylene suture of the stump.

\section{Discussion}

Thanks to Claude Franceschi's diagnostic experience with echo color Doppler ultrasound, it was possible to highlight anatomical parietal points of passage of refluxes from the veins of the pelvis to the

Figure 3. Valsalva reflux from the left perineal point (posterior labial vein). The vulvar tissue is the black dishomogeneous part in the lower half of the picture. The white dashed line represent the position of the labia minora frenulum.

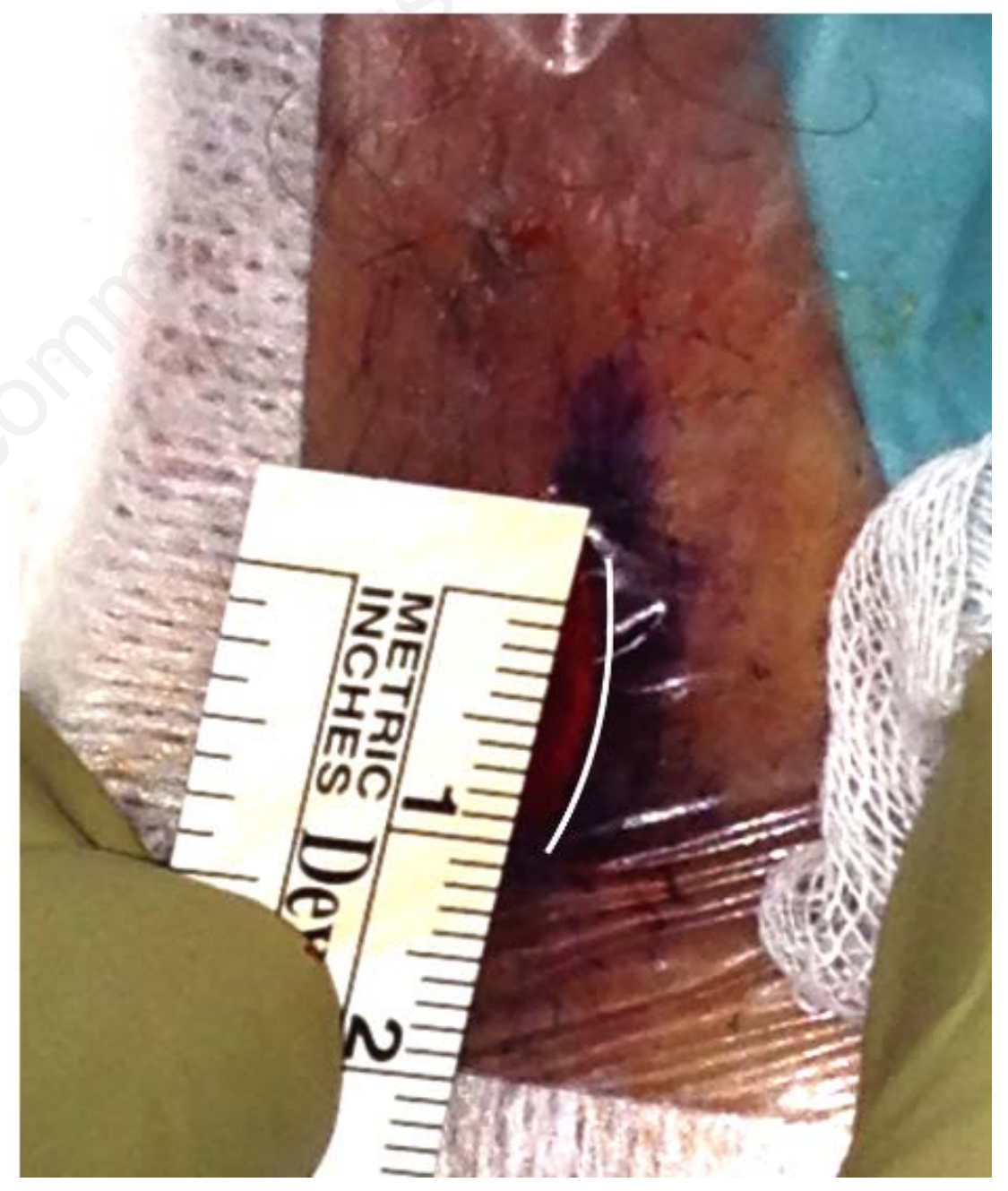

Figure 4. Minimally invasive surgical access to the fascia hole of the posterior labial vein. 
lower limbs. The experience in the anatomical exploration with ultrasounds, supported by the re-evaluation of the knowledge of human anatomy, has allowed us to propose a minimally invasive therapeutic approach, under local anesthesia, of the pelvic refluxes that feed varicose veins of the lower limbs based on the disconnection of venous collectors in well-defined anatomical areas, constant and easy to access surgically, but not perfectly known by both radiologists and vascular or general surgeons. This minimally invasive therapeutic strategy consists of three different phases: diagnosis, preoperative marking and surgical treatment. The diagnostic phase allows the creation of precise hemodynamic maps in order to detect the escape points, escape routes and reentry points of each shunt. The preoperative skin marking, besides allowing a check of the accuracy of cartography, identifies the exact site of the surgical access and allows a pre-operative visual assessment thanks to the ultrasounds, useful for the surgical dissection, and finally the surgical implementation of the disconnections at the level of the pelvic escape points. In the absence of pelvic congestion syndrome the interruption of the reflux from the pelvis to the lower limbs is without negative consequences on pelvic drainage, which will continue to occur regularly as in the physiological conditions according to the pressure gradient existing with the right atrium. The primary objective of this study was to verify the elimination of reflux at the level of the treated pelvic escape points and the secondary objective was to verify the stability of the result over time.

It should be noted that there were almost no complications in the procedures performed, except for the only complicative event detected of postoperative bleeding during the correction of a groin point, which was followed by a modification of the technique of ligation of the stumps of the venous plexus of the round ligament aimed at making the section and ligation very reliable. Unlike our experience in the surgical treatment of pelvic escape points, the endovascular method is burdened with well-known complications. These are mechanical complications, the consequence of puncturing a central vein (haematomas, pneumothorax, arthymia, gas embolism, arterial puncture), and other adverse events typical of the method, for instance the migration of coils, or in case of use of other agents such as mousse or glue, injections, the migration even in districts other than the desired one. Another point to be clarified about endovascular procedures is the possible effect on fertility reported in the literature although not confirmed in the experi-

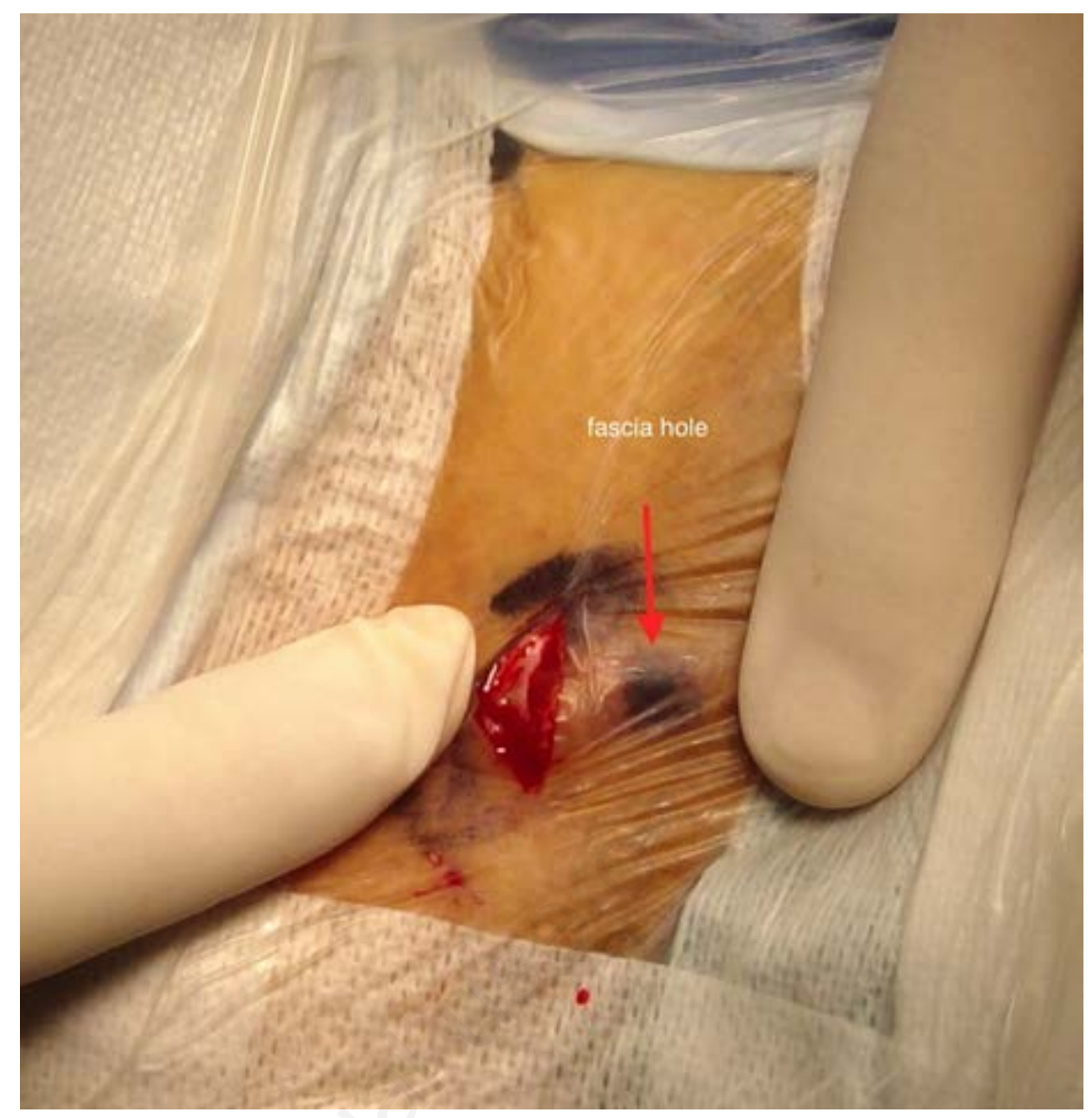

Figure 5. Comparison between the skin incision and the skin mark of the fascia hole of the posterior labial vein.

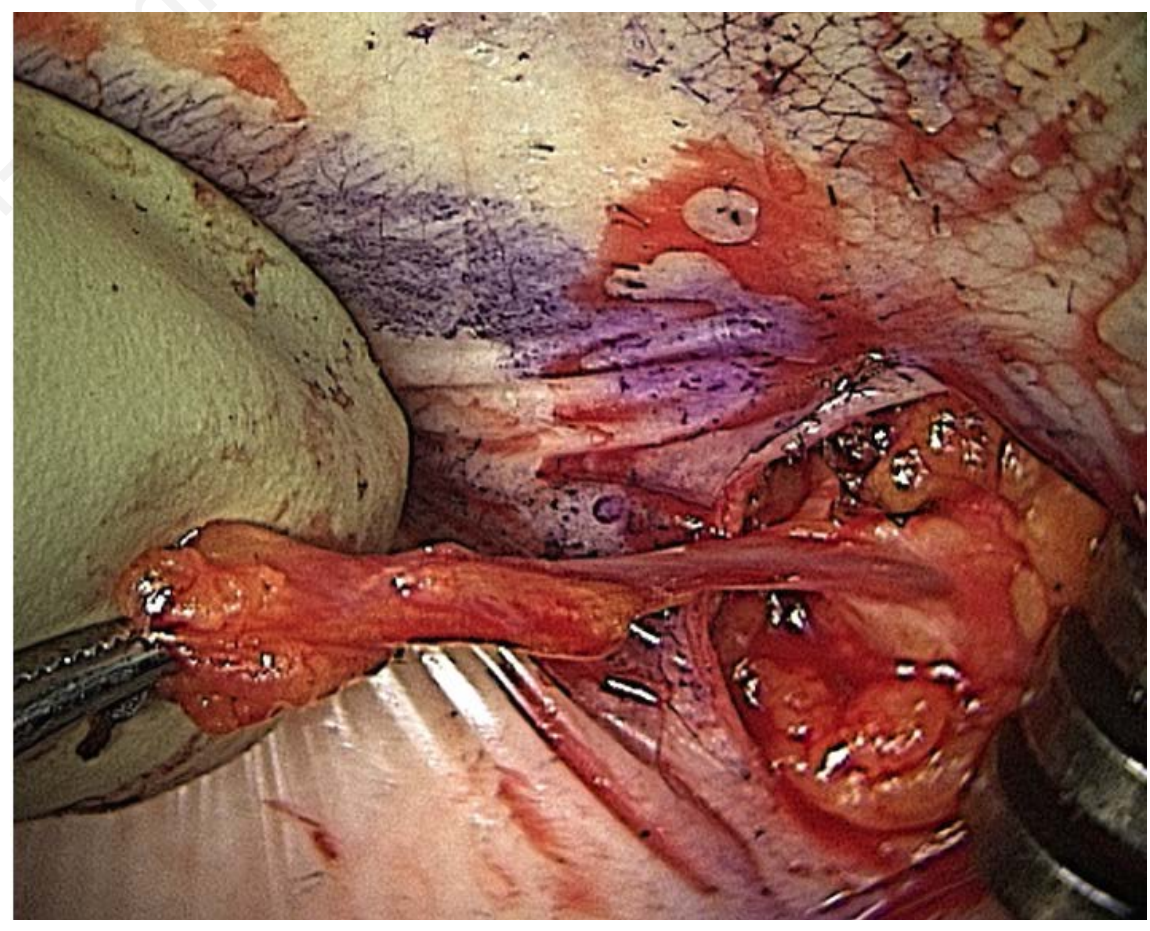

Figure 6. The posterior labial vein crossing the vulvar fascia hole to reach the vulvar venous plexuses. 
ence of some authors. This study shows that echo-guided minimally-invasive surgery of pelvic leaks responsible for vulvar and/or lower limbs varicose veins is reliable and durable. This study suggests that pelvic varicose embolization prior PLP reflux ablation is not necessary and indicated only in case of resistant PLP reflux or when PLP is associated with pelvic congestion syndrome, according to others. ${ }^{24}$ On the other hand, prior pelvic vein embolization leaves behind a PLP reflux, even if reduced, which needs complementary superficial treatment. ${ }^{25}$ Sclerosing agents and foam are also used to treat the PLP by injecting the extra pelvic veins. No long-term study has been published so far. ${ }^{26-30}$ This study does not report the additional CHIVA disconnections nor their specific outcomes because they do not determine the persistence of the PLP treatment. The low rate of failures may be due to the surgical technique: vein division-ligation and fascia/superficial inguinal channel suture with no absorbable monofilament suture, which is supposed to avoid both collateral reflux and neoangiogenesis (inflammatory reaction to absorbable suture), as well as the accurate ultrasound detection of the leaking points that allows for a very minimally invasive operation. In order to reduce possible mistake in case of PP treatment an accurate DUS control of the skin marking is necessary in the operative theatre with the patients in lithotomy position.

\section{Conclusions}

This study shows that echo-guided minimally-invasive surgery of pelvic leaks responsible for vulvar and/or lower limbs varicose veins is reliable and durable.

This study also suggests that pelvic varicose embolization prior to PLP reflux ablation is not necessary. The accurate ultrasound assessment of each specific pelvic leak as well as a special minimally invasive surgical technique (ambulatory patient, local anesthesia, non-absorbable suture of vein stumps and fascias, low surgical risk and low percentage of complications) seems to be the key to satisfactory outcomes.
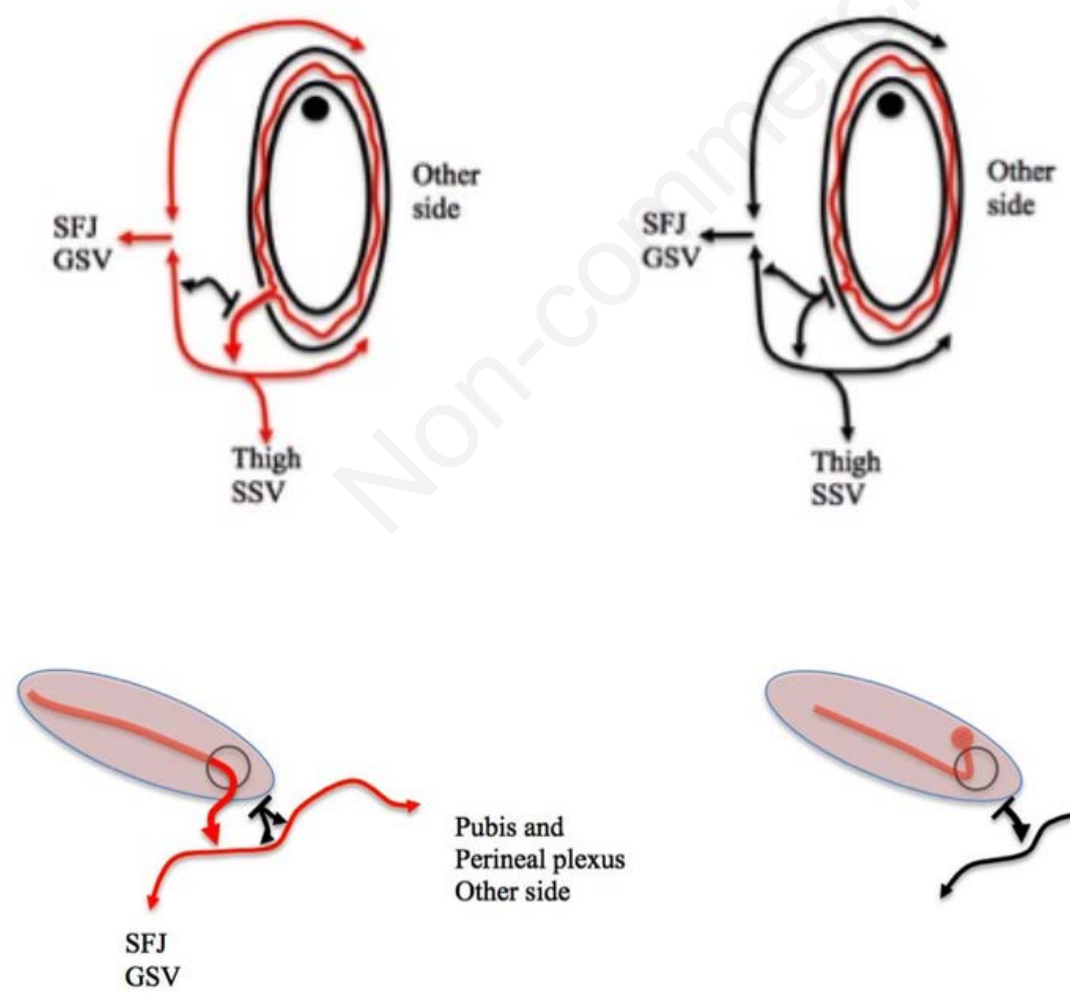

Pubis and Perineal plexus Other side

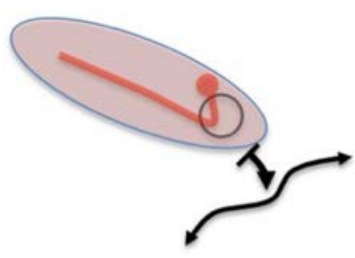

SFJ

Figure 7. Ligation of leak points. The perineal point (upper part of the figure) and inguinal leak points (lower part of the figure) act as perforating veins. Remote disconnection as represented on the left side, invariably fails either immediately or secondarily because of the presence of many branches and anastomoses. On the right side of the figure the correct level of disconnection is shown.

\section{References}

1. Franceschi C, Bahnini A. Points de fuite pelviens viscéraux et varices des membres inférieurs. Phlébologie 2004; 57:37-42.

2. Franceschi C, Bahnini A. Treatment of lower extremity venous insufficiency due to pelvic leak points in women. Ann Vasc Surg 2005;19:284-8.

3. Franceschi C. Anatomie fonctionnelle et diagnostic des points de fuite bulboclitoridiens chez la femme (point C). J Mal Vasc 2008;33:42.

4. Franceschi C, Zamboni P. Principles of venous haemodynamics. New York: Nova Science; 2008.

5. Ricci S. Phlebology in 21st century. Veins and Lymphatics 2014;3:2268.

6. Jiang P, Van Rij AM, Christie RA, et al. Non-saphenofemoral venous reflux in the groin in patients with varicose veins. Eur J Vasc Endovasc Surg 2001; 21:550-7.

7. Barros FS, Perez JMG, Zandonade E, et al. Evaluation of pelvic varicose veins using color Doppler ultrasound: comparison of results obtained with ultrasound of the lower limbs, transvaginal ultrasound, and phlebography. J Vasc Bras 2010;9:15-23.

8. García-Gimeno M, RodríguezCamarero S, Tagarro-Villalba S, et al. Duplex mapping of 2036 primary varicose veins. J Vasc Surg 2009;49:681-9.

9. Labropoulos N, Tiongson J, Pryor P, et al. Nonsaphenous superficial vein reflux. J Vasc Surg 2001;34:872-7.

10. Perrin N, Labropoulos N, Leon L. Presentation of the patient with recurrent varices after surgery (REVAS). J Vasc Surg 2006;43:327-34.

11. Reich-Schupke S, Mendoza E, Dörler, M. Stücker M. Frequency of refluxive tributaries of the junction region in the groin in patients with recurrent varicose veins of the thigh. Phlebologie 2016;45:149-53.

12. Hüffer A. Lehrbuch der topographischen anatomie. Berlin, Heidelberg, New York: Springer-Verlag; 1969.

13. Sala L, Bruni C. Trattato di anatomia umana. vol. 4. Milano: Ed. Vallardi; 1932.

14. Fusari R, Bruni AC. Trattato di anatomia umana topografica. Torino: Ed. UTET; 1936.

15. Pernkopf E. Atlas of topographical and applied human anatomy. Vol. II; 222223.

16. Leal Monedero J, Zubicoa Ezpeleta S, Castro C, et al. Embolization treatment of recurrent varices of pelvic origin. 
Phlebology 2006;21:3-11.

17. Greiner M. Varices des membres inférieurs d'origine pelvienne: traitement et résultats à long terme. J Mal Vasc 2006;31:C2, 26.

18. Criado E, Juan J, Fontcuberta J, Escribano MJ. Haemodynamic surgery for varicose veins: rationale, and anatomic and haemodynamic basis. Phlebology 2003;18:158-66.

19. Delfrate R, Bricchi M, Franceschi C, Goldoni M. Multiple ligation of the proximal greater saphenous vein in the CHIVA treatment of primary varicose veins. Veins and Lymphatics 2014;3:1922.

20. Franceschi C. Theorie et practique de la Cure Conservatrice et Hemodynamique de l'Insuffisance Veineuse en Ambulatroire. Precy-sous-thil, France: Edition de 1'Armançon; 1988.
21. Franceschi C, Cappelli M, Ermini S, et al. CHIVA: hemodynamic concept, strategy and results. Int Angiol 2016;35:8-30.

22. Delfrate R. A new diagnostic approach to varicose veins: haemodynamic evaluation and treatment. Folgaria (TN): Lorena Dioni Publishing; 2014.

23. Zamboni P, Mendoza E, Gianesini S. Saphenous vein-sparing strategies in Chronic venous disease. Berlin: Springer International Publishing; 2018.

24. Rabe E, Pannier F. Embolization is not essential in the treatment of leg varices due to pelvic venous insufficiency. Phlebology 2015;30:86-8.

25. Lopez AJ. Female pelvic vein embolization: Indications, Techiques and Outcomes. Cardiovasc Intervent Radiol 2015;38:806-20.
26. Kim AS, Greyling LA, Davis LS. Vulvar varicosities: a review. Dermatol Surg 2017;43:351-6.

27. Sofler M, Hayes M, Smith C. Central venous catheterization training: current perspectives on the role of simulation. Adv Med Educ Prat 2018;9:395-403.

28. Parienti JJ. Intravascular complications of central venous catheterization by insertion site. N Engl J Med 2015;373: 1220-9.

29. McGee DC, Gould MK. Preventing complications of central venous catheterization. N Engl J Med 2003; 348:1123-33.

30. Rando K. Ultrasound-guided internal jugular vein catheterization: a randomized controlled trial. Heart Lung Vessel 2014;6:13-23. 